\title{
CR STRUCTURES ON OPEN MANIFOLDS
}

\author{
HOWARD JACOBOWITZ AND PETER LANDWEBER
}

\begin{abstract}
We show that the vanishing of the higher dimensional homology groups of a manifold ensures that every almost CR structure of codimension $k$ may be homotoped to a $\mathrm{CR}$ structure. This result is proved by adapting a method due to Haefliger used to study foliations (and previously applied to study the relation between almost complex and complex structures on manifolds) to the case of (almost) CR structures on open manifolds.
\end{abstract}

\section{INTRODUCTION}

An almost complex structure on an even dimensional smooth manifold $M$ is a smooth linear vector bundle map $J$ on the tangent bundle of $M$ satisfying $J^{2}=$ $-I$. We pass to the complexified tangent bundle and denote by $B$ the bundle of eigenspaces of $J$ with eigenvalue $+i$. So the elements of $B$ correspond to the anti-holomorphic vectors. We have

$$
B \cap \bar{B}=\{0\} \text { and } B \oplus \bar{B}=\mathbf{C} \otimes T(M) .
$$

These steps are reversible, and in this paper we shall view an almost complex structure as being such a subbundle of the complexified tangent bundle.

Being given an almost complex structure $B$ on $M$, one says that $B$ defines a complex structure on $M$ if in an open neighborhood of each point complex coordinates may be introduced so that $B$ is spanned by the set of vectors

$$
\frac{\partial}{\partial \bar{z}_{j}}, \quad j=1, \ldots, \frac{1}{2} \operatorname{dim} M
$$

The Newlander-Nirenberg Theorem [19] asserts that $B$ defines a complex structure if and only if $B$ is involutive, by which we mean that the bracket of two smooth sections of $B$ defined on an open subset of $M$ is also a section of $B$, i.e., the space $\mathcal{B}$ of smooth vector fields with values in $B$ satisfies

$$
[\mathcal{B}, \mathcal{B}] \subset \mathcal{B} .
$$

More generally, any subbundle of $\mathbf{C} \otimes T(M)$ is said to be involutive if this condition is satisfied.

This, of course, is reminiscent of the Frobenius condition for foliations. It is to be expected that various techniques developed in the theory of foliations would have analogues whenever subbundles of $\mathbf{C} \otimes T(M)$ are involutive. In particular, we ask: When does $M$ admit an involutive subbundle $B$ which satisfies $B \cap \bar{B}=\{0\}$ and has a specified complex dimension? For almost complex structures these techniques do extend, see [1], 14]. Using somewhat different techniques from foliation theory,

Date: May 7, 2014.

2010 Mathematics Subject Classification. Primary 32V05; Secondary 55S35.

Key words and phrases. almost complex, almost CR, Haefliger structure, Gromov's h-principle. 
we provide an answer for almost $\mathrm{CR}$ structures on manifolds having vanishing homology in high dimensions.

There are two basic steps to our argument. Following Haefliger's work in foliation theory, we broaden the definition of an almost CR structure to a more flexible category (in Section 3) in order to apply (in Section 7) homotopy theory to study the existence of liftings of a continuous map $X \rightarrow B$ to a map $X \rightarrow E$, where $E \rightarrow B$ is a given fibration. This is used to construct a manifold $X$, foliated by complex manifolds, and a map $f: \mathbf{C} \otimes T(M) \rightarrow T^{1,0}(X)$ with appropriate properties. We then use Gromov's h-principle to prove an analogue of the GromovPhillips Theorem in order to replace $f$ by a map $F: M \rightarrow X$ whose differential has these properties (specifically, Conditions 1 and 2 of Theorem 4.1) and thereby induces on $M$ the desired CR structure.

For convenience, here is the main result. Definitions are in the next section.

Theorem 1.1. If

$$
H_{p}\left(M^{2 n+k} ; \mathbf{Z}\right)=0 \text { for } p \geq n+k+1
$$

then every smooth almost $C R$ structure of codimension $k$ on $M$ is homotopic to a $C^{\omega}$ CR structure of codimension k. In particular, every $C^{\infty}$ CR structure may be deformed to a $C^{\omega}$ CR structure.

\section{THE BASICS}

Let $M$ be a manifold of dimension $2 n+k$ with $k>0$. Let $N$ stand for some unspecified manifold of the same dimension, which will often be just an open ball in $\mathbf{R}^{2 n+k}$. All manifolds, bundles, and maps are of class $C^{\infty}$, and all manifolds are paracompact, unless otherwise indicated. (It is likely that the results discussed here also hold with only minimal smoothness assumptions.) All open sets which are introduced to state local results are taken to be connected and sufficiently small.

An almost CR structure of codimension $k$ : on $M$ is a complex subbundle $B \subset \mathbf{C} \otimes T(M)$ of complex dimension $n$ that satisfies $B \cap \bar{B}=\{0\}$.

A CR structure of codimension $k$ : is an almost CR structure $B$ of codimension $k$ that in addition is involutive.

A generic CR immersion: is an immersion $f$ of $M$ into a complex manifold $X$ of complex dimension $n+k$ such that $(\mathbf{C} \otimes T(M)) \cap f^{*} T^{0,1}(X)$ has complex dimension $n$ at all points of $M$. We set $B=(\mathbf{C} \otimes T(M)) \cap$ $f^{*} T^{0,1}(X)$ and observe that such an immersion induces a CR structure on $M$. Conversely, when the $\mathrm{CR}$ structure $B$ is given we require that the immersion induces this CR structure.

Remark 1. Results similar to ours should hold for a large class of involutive systems.

Two things are well known:

- Not all CR structures can be obtained by immersions into complex manifolds. That is, there exist smooth CR structures that are not induced by local embeddings into $\mathbf{C}^{n+k}$. Nirenberg [20, page 13] gave the first examples of such non-realizable $\mathrm{CR}$ structures.

- Real analytic CR structures are obtained by immersions into $\mathbf{C}^{n+k}$ and there is even an associated uniqueness result. For the convenience of the reader we now state and prove this result. 
Lemma 2.1. If $M$ has a $C^{\omega} C R$ structure $B$ of codimension $k$ then there exists an open covering

$$
M=\bigcup_{j} \mathcal{O}_{j}
$$

and $C^{\omega}$ generic $C R$ embeddings

$$
f_{j}: \mathcal{O}_{j} \rightarrow \mathbf{C}^{n+k} \text {. }
$$

Further, for each pair $(i, j)$ with $\mathcal{O}_{i} \cap \mathcal{O}_{j} \neq \emptyset$ there exists an open set $U_{i j}$ containing $f_{i}\left(\mathcal{O}_{i} \cap \mathcal{O}_{j}\right)$ and a biholomorphism $\gamma_{i j}: U_{j i} \rightarrow U_{i j}$ with

$$
f_{i}=\gamma_{i j} \circ f_{j} \text { on } \mathcal{O}_{i} \cap \mathcal{O}_{j} .
$$

Proof. We reduce this lemma to the corresponding result for $C^{\omega}$ almost complex manifolds by applying the following theorem.

Theorem 2.1 ([6], 17]). Let $Q_{1}, \ldots, Q_{N}$ be real analytic vector fields in a neighborhood of the origin in $\mathbf{R}^{2 N}$ satisfying

(1) $Q_{1}, \ldots, Q_{N}, \overline{Q_{1}}, \ldots \overline{Q_{N}}$ span $\mathbf{C} \otimes \mathbf{R}^{2 N}$

(2) $\left[Q_{i}, Q_{j}\right]$ is in the linear span of $\left\{Q_{1}, \ldots, Q_{N}\right\} \quad$ for $1 \leq i, j \leq N$.

Then there exist complex coordinates $\left\{z_{1}, \ldots, z_{N}\right\}$ on a possibly smaller neighborhood of the origin such that

$$
\left\{Q_{1}, \ldots, Q_{N}\right\}=\left\{\partial_{\overline{z_{1}}}, \ldots, \partial_{\overline{z_{N}}}\right\} .
$$

To use this theorem to prove the lemma, let $L_{1}, \ldots, L_{n}$ be real analytic and span the CR bundle $B$ near some point $p \in M$. Choose coordinates

$$
x_{1}, \ldots, x_{2 n}, t_{1}, \ldots, t_{k}
$$

so that

span $B \oplus \bar{B}$ at $p$. First write

$$
\partial x_{1}, \ldots, \partial x_{2 n}
$$

$$
L_{j}=\sum_{p=1}^{2 n} \alpha_{j p}(x, t) \partial_{x_{k}}+\sum_{m=1}^{k} \beta_{j m}(x, t) \partial_{t_{m}}, \quad j=1, \ldots, n,
$$

with coefficients $\alpha$ and $\beta$ in $C^{\omega}$ and then extend each vector field to $\mathbf{R}^{2 n+2 k}$ by

$$
L_{j}=\sum_{p=1}^{2 n} \alpha_{j p}(x, t+i s) \partial_{x_{k}}+\sum_{m=1}^{k} \beta_{j m}(x, t+i s) \partial_{t_{m}} .
$$

Let $\widetilde{B}$ be the linear span of these extended vector fields together with the vector fields

$$
\partial_{t_{m}}+i \partial_{s_{m}}, \quad m=1, \ldots, k
$$

Then, using the fact that the coefficients are holomorphic in $t+i s, \widetilde{B}$ is involutive and so the above Theorem applies:

$$
\widetilde{B}=\left\{\partial_{\overline{z_{1}}}, \ldots, \partial_{\overline{z_{n+k}}}\right\}
$$

This gives us an embedding of a neighborhood of $p$ in $M$ into $\mathbf{C}^{n+k}$ with

$$
B=(\mathbf{C} \otimes T(M)) \cap T^{0,1}\left(\mathbf{C}^{n+k}\right) .
$$

Thus the embedding is a generic CR embedding. 
To find $\gamma_{i j}$ we assume that $\mathcal{O}_{i} \cap \mathcal{O}_{j}$ is not empty. Thus we have a diffeomorphism

$$
\Phi: f_{i}\left(\mathcal{O}_{i} \cap \mathcal{O}_{j}\right) \rightarrow f_{j}\left(\mathcal{O}_{i} \cap \mathcal{O}_{j}\right)
$$

with $\Phi_{*} B=B$. This implies that each component of $\Phi$ is a $C^{\omega}$ CR function. That is, for each local section $L$ of $B$ we have

$$
L \Phi_{j}=0 .
$$

Such a function on a generic submanifold of a complex manifold is the restriction of a holomorphic function. See, for instance [2, page 29, Corollary 1.7.13]. We note for later use that the proof of this Corollary also shows that the extension is unique.

Let $\gamma_{i j}$ denote this extension. If $\gamma_{i j}$ were not a biholomorphism in some neighborhood of $f_{i}\left(\mathcal{O}_{i} \cap \mathcal{O}_{j}\right)$ then there would be a nonzero vector

$$
v=\sum_{m=1}^{n+k} \alpha_{m} \partial z_{m}
$$

annihilated by $d \gamma_{i j}$ at some $p \in f_{i}\left(\mathcal{O}_{i} \cap \mathcal{O}_{j}\right)$. Since the submanifold is CR generic, $v=T-i J T$ for some $\left.T \in T M\right|_{p}$. But since $\gamma_{i j}$ has been extended holomorphically

$$
\begin{aligned}
d \gamma v & =d \gamma T-i d \gamma J T \\
& =d \gamma T-i J(d \gamma T) .
\end{aligned}
$$

So if $\gamma_{i j}$ is not a biholomorphism, $d \gamma_{i j}$ annihilates a nonzero vector in $T M$ and this is impossible because $\gamma_{i j}$ is a real diffeomorphism on $f_{i}\left(\mathcal{O}_{i} \cap \mathcal{O}_{j}\right)$.

We note that

$$
\gamma_{i k}=\gamma_{i j} \circ \gamma_{j k}
$$

on the domain $f_{k}\left(\mathcal{O}_{i} \cap \mathcal{O}_{j} \cap \mathcal{O}_{k}\right)$. To see this let $p \in f_{k}\left(\mathcal{O}_{i} \cap \mathcal{O}_{j} \cap \mathcal{O}_{k}\right)$. Then there exists some $x \in \mathcal{O}_{i} \cap \mathcal{O}_{j} \cap \mathcal{O}_{k}$ with $f_{k}(x)=p$. Thus

$$
\gamma_{i k}(p)=\gamma_{i k} \circ f_{k}(x)=f_{i}(x) \text {. }
$$

Further,

$$
\gamma_{i j} \circ \gamma_{j k}(p)=\gamma_{i j} \gamma_{j k} \circ f_{k}(x)=\gamma_{i j} \circ f_{j}(x)=f_{i}(x)=\gamma_{i k}(p) .
$$

By the uniqueness of extensions of holomorphic functions off of generic submanifolds, as referenced in the previous proof, the fact that

$$
\gamma_{i k}=\gamma_{i j} \circ \gamma_{j k}
$$

holds on $f_{k}\left(\mathcal{O}_{i} \cap \mathcal{O}_{j} \cap \mathcal{O}_{k}\right)$ implies that it holds wherever it makes sense.

\section{Haefliger StruCtures}

Following a well-known procedure in foliation theory, as in [15] or [18 we generalize the objects in Lemma 2.1 by dropping the requirements that the maps are smooth (indeed analytic) and are embeddings. As a reminder, we consider only paracompact manifolds. Let $A$ be some index set.

Definition 1. A Haefliger CR structure of codimension $k$ on $M^{2 n+k}$ consists of

- An open covering $M=\bigcup_{j} \mathcal{O}_{j}, j \in A$,

- continuous maps $f_{j}: \mathcal{O}_{j} \rightarrow \mathbf{C}^{n+k}$,

- local biholomorphisms $\gamma_{i j}$ of $\mathbf{C}^{n+k}$ defined for each pair $(i, j)$ such that $\mathcal{O}_{i} \cap \mathcal{O}_{j} \neq \emptyset$ satisfying:

(1) $\gamma_{i k}=\gamma_{i j} \circ \gamma_{j k}$ at all points where both sides are defined, and 
(2) $f_{i}=\gamma_{i j} \circ f_{j}$ on $\mathcal{O}_{i} \cap \mathcal{O}_{j}$.

Lemma 2.1 shows that a $C^{\omega} \mathrm{CR}$ structure admits a Haefliger CR structure of a special kind. Namely, each $f_{j}$ is an embedding.

Let $A_{i j}$ and $B_{i j}$ denote, respectively, the domain and codomain of $\gamma_{i j}$. So $\gamma_{i j}$ induces a bijection $\gamma_{i j *}: T^{1,0}\left(A_{i j}\right) \rightarrow T^{1,0}\left(B_{i j}\right)$. We may restrict $\gamma_{i j *}$ to the image of $\mathcal{O}_{i} \cap \mathcal{O}_{j}$ and then pull back to $M$ to obtain transition functions $g_{i j}$ that patch $\mathcal{O}_{i} \times \mathbf{C}^{n+k}$ to $\mathcal{O}_{j} \times \mathbf{C}^{n+k}$ over $\mathcal{O}_{i} \cap \mathcal{O}_{j}$, and so determine a vector bundle:

Definition 2. The normal bundle $\nu$ of a Haefliger $C R$ structure is the complex $(n+k)$-dimensional vector bundle over $M$ with transition functions $g_{i j}$.

Theorem 3.1. If $B$ is a $C^{\omega} C R$ structure then $(\mathbf{C} \otimes T(M)) / B$ is isomorphic to the normal bundle of a Haefliger CR structure.

Proof. By Lemma 2.1, $M$ admits a Haefliger CR structure. Each $f_{i}$ is a generic CR map, so we have over $\mathcal{O}_{i}$,

$$
B \cong f_{i *} B=(\mathbf{C} \otimes T(M)) \cap T^{0,1} .
$$

We claim this implies

$$
\left.((\mathbf{C} \otimes T(M)) / B)\right|_{\mathcal{O}_{i}} \cong f_{i}^{*} T^{1,0} .
$$

To see this consider the map $\mathbf{C} \otimes T(M) \rightarrow T^{1,0}$ given by, again over $\mathcal{O}_{i}$,

$$
\Phi: Y \mapsto f_{i *} Y-i J f_{i *} Y
$$

where $J$ is the standard anti-involution on $\mathbf{C}^{n+k}$. Note that $\Phi(Y)=0$ if and only if

$$
Y \in(\mathbf{C} \otimes T(M)) \cap T^{0,1}=f_{i *} B .
$$

Thus the kernel of $\Phi$ has complex dimension $n$, which implies that $\Phi$ is surjective. Then (3.1) follows.

But the transition functions for $\left.T^{1,0}\right|_{f_{i}(M)}$ are just the derivative maps $g_{i j}$. Thus $(\mathbf{C} \otimes T(M)) / B$ is isomorphic to the normal bundle of this Haefliger CR structure.

The same conclusion holds if $B$ is homotopic (even through $2 n$-plane bundles which may not be almost $\mathrm{CR}$ ) to a $C^{\omega} \mathrm{CR}$ structure.

We can now state the first of two basic steps in the proof of our main theorem. Here there is no restriction on the manifold. But for the second step, Theorem 7.1 the topology of the manifold is restricted. In particular, $M$ needs to be open.

Theorem 3.2. Let $B$ be a smooth almost $C R$ structure of codimension $k$ on $M^{2 n+k}$. If $(\mathbf{C} \otimes T(M)) / B$ is isomorphic to the normal bundle of a Haefliger CR structure then $B$ is homotopic through almost $C R$ structures of codimension $k$ to a $C^{\omega} C R$ structure.

The next two sections contain the proof of this theorem.

Corollary 1. Let $B$ be a smooth $C R$ structure. Then $B$ is homotopic to a $C^{\omega} C R$ structure if and only if $(\mathbf{C} \otimes T(M)) / B$ is isomorphic to the normal bundle of a Haefliger CR structure.

It is not known if every smooth CR structure is homotopic to a $C^{\omega} \mathrm{CR}$ structure. It is possible that this is true on some manifolds and false on others. (It is certainly true locally.) We shall see in Theorem 8.1 that it is true provided $M$ satisfies some topological restrictions. 


\section{The CR EMBedding}

In this section we reduce the proof of Theorem 3.2 to an h-principle argument which is then provided in the next section.

Lemma 4.1. Given a Haefliger $C R$ structure on $M^{2 n+k}$ there exists a manifold $X$ of real dimension $4 n+3 k$ and an embedding $\iota: M \rightarrow X$ such that

(1) $X$ is a fiber bundle over $M$ with complex structure on the fibers.

(2) $X$ admits a foliation $\mathcal{F}^{2 n+k}$ transverse to the fibers.

(3) The normal bundle $\nu$ of the Haefliger structure is isomorphic to

$$
\iota^{*} T(X) / T(M) .
$$

Proof. Let $M$ have local charts

$$
\phi_{i}: \mathcal{O}_{i} \rightarrow \mathbf{R}^{2 n+k} .
$$

Set $\Omega$ equal to the disjoint union

$$
\Omega=\bigsqcup_{i}\left(\phi_{i}\left(\mathcal{O}_{i}\right) \times \mathbf{C}^{n+k}\right),
$$

and define an equivalence relation on $\Omega$ by setting

$$
\left(x_{i}, y_{i}\right) \sim\left(x_{j}, y_{j}\right)
$$

whenever

$$
\begin{aligned}
x_{j} & =\phi_{j} \phi_{i}^{-1}\left(x_{i}\right) \quad \text { and } \\
y_{j} & =\gamma_{j i}\left(y_{i}\right) .
\end{aligned}
$$

Here $\gamma_{j i}$ are the local biholomorphisms of the Haefliger structure. Denote the quotient space, with the quotient topology, by $Z$. Note that the projection map

$$
\Omega \rightarrow Z
$$

is an open map and that the projection map

$$
\pi: Z \rightarrow M
$$

is well-defined. The multi-valued map of $M$ into $\Omega$

$$
p \mapsto \bigsqcup_{i}\left(p, f_{i}(p)\right)
$$

for $p \in \mathcal{O}_{i}$ passes down to a well-defined map

$$
\iota: M \rightarrow Z \text {. }
$$

Clearly, $\pi \circ \iota$ is the identity.

We shall see that $Z$ is Hausdorff near $\iota(M)$. This provides the manifold $X$. To show that $Z$ is Hausdorff near the image of $M$ let $a=\left[\left(x_{i}, y_{i}\right)\right]$ and $b=\left[\left(x_{j}, y_{j}\right)\right]$ be points of $Z$. If $x_{j} \neq \phi_{j} \phi_{i}^{-1}\left(x_{i}\right)$, then we can separate $a$ and $b$ by disjoint open sets. So now let $a=\left[\left(x_{i}, y_{i}\right)\right]$ and $b=\left[\left(\phi_{j} \phi_{i}^{-1}\left(x_{i}\right), y_{j}\right)\right]$. We may assume (by staying close enough to $\iota(M))$ that $y_{i}$ is in the domain of $\gamma_{j i}$. Thus if $y_{j} \neq \gamma_{j i}\left(y_{i}\right)$ we may again separate $a$ and $b$ while if $y_{j}=\gamma_{j i}\left(y_{i}\right)$ then $a=b$.

Note that the transition functions for $X$ are

$$
\psi_{j i}(x, y)=\left(\phi_{j} \phi_{i}^{-1}(x), \gamma_{j i}(y)\right)
$$

for $x \in \phi_{i}\left(\mathcal{O}_{i} \cap \mathcal{O}_{j}\right)$ and $y$ close enough to $\gamma_{j i}\left(f_{i}\right)$. This implies that the two natural foliations on $\Omega$ corresponding to each factor in $\phi_{i}\left(\mathcal{O}_{i}\right) \times \mathbf{C}^{n+k}$ pass down to $X$. 
In particular, the complex structure on each fiber $\pi^{-1}(p)$ is well-defined. (Here the map $\pi$ of (4.3) is restricted to $X \subset Z$.) Thus (11) and (2) of the Lemma hold. Further, the transition functions for $T(X)$ are

$$
\psi_{j i *}(x, y)=\left(\left(\phi_{j} \phi_{i}^{-1}\right)_{*}(x), \gamma_{j i *}(y)\right) .
$$

Restricting to $\iota(M)$ we have

$$
\begin{aligned}
\psi_{j i *}(x) & =\left(\left(\phi_{j} \phi_{i}^{-1}\right)_{*}(x), \gamma_{j i *}\left(f_{i}(x)\right)\right) \\
& =\left(\left(\phi_{J} \phi_{i}^{-1}\right)_{*}, g_{j i}\left(f_{i}(x)\right)\right) .
\end{aligned}
$$

So the transition functions for the normal bundle of $\iota(M) \subset X$ are the same as those for $\nu$, the normal bundle of the Haefliger structure. This establishes (3).

To explain what comes next, we review a well-known procedure in foliation theory. Let $\mathcal{M}$ have a foliation $\mathcal{F}$ of codimension $q$. If $\Phi: M \rightarrow \mathcal{M}$ is transverse to the leaves of $\mathcal{F}$, in the sense that $\Phi_{*} T(M)$ and $T(\mathcal{F})$ together span $T(\mathcal{M})$ at each point of the image of $M$, then, as is easily seen, $\mathcal{F}$ induces a foliation on $M$, of the same codimension. By the Gromov-Phillips Theorem (see, for instance, [18, Section 4, pp. 293-297] when $M$ is open, it is enough to have a bundle map

$$
\Psi: T(M) \rightarrow T(\mathcal{M})
$$

satisfying the tranversality condition, for then the base map of $\Psi$ may be deformed to a transverse map $\Phi$.

We rephrase this as: An open manifold $M$ admits a codimension- $q$ foliation provided there exists a vector bundle map $\Psi$ such that the composition

$$
T(M) \stackrel{\Psi}{\longrightarrow} T(\mathcal{M}) \longrightarrow T(\mathcal{M}) / T(\mathcal{F})
$$

is surjective, where $\mathcal{F}$ is a codimension- $q$ foliation of $\mathcal{M}$.

To state the modification of the Gromov-Phillips Theorem we use, we first need some definitions. Let $\pi: X \rightarrow M$ be the projection provided by the Lemma. Recall that each fiber $\pi^{-1}(p)$ is a complex manifold. Set

$$
T_{f}=\bigcup_{p \in M} T\left(\pi^{-1}(p)\right) \subset T(X)
$$

and

$$
T_{f}^{1,0}=\bigcup_{p \in M} T^{1,0}\left(\pi^{-1}(p)\right) \subset \mathbf{C} \otimes T(X) .
$$

The subscript is to remind us that we are looking at tangents to the fiber. The form of the transition functions for $X$, see (4.4), shows that the map

$$
\mu: \mathbf{C} \otimes T(X) \rightarrow T_{f}^{1,0}
$$

is well-defined.

Although the following is patterned upon the Gromov-Phillips Theorem, the restriction to open manifolds is not necessary here.

Theorem 4.1. If there exists a surjective bundle map

$$
\Psi:\left.\mathbf{C} \otimes T(M) \rightarrow T_{f}^{1,0}\right|_{M}
$$

then there exists a smooth map

$$
F: M \rightarrow X
$$

such that 
(1) The composition

$$
\mu F_{*}: \mathbf{C} \otimes T(M) \rightarrow \mathbf{C} \otimes T(X) \rightarrow T_{f}^{1,0}
$$

is surjective.

(2) $\mu_{*} F_{*}$ is injective when restricted to $T(M)$.

(3) $B_{1}=\operatorname{ker} \mu F_{*}$ is homotopic to $B=\operatorname{ker} \Psi$.

This is the heart of the proof of Theorem 3.2. We prove it in the next section by means of Gromov's h-principle. Here let us first see how it implies the conclusion of Theorem 3.2 and then how we use the hypothesis

$$
(\mathbf{C} \otimes T(M)) / B \cong \nu
$$

to obtain the surjective map

$$
\Psi:\left.\mathbf{C} \otimes T(M) \rightarrow T_{f}^{1,0}\right|_{M}
$$

We first show that $B_{1}$ defines a CR structure.

Lemma 4.2. The bundle $B_{1}$ is involutive.

Proof. It suffices to assume that $a$ and $b$ are local sections of $B_{1}$ and to show that

$$
\mu F_{*}[a, b]=0 \text {. }
$$

We work in local coordinates. So we write

$$
F(x)=(f(x), y(x)) \in \phi_{i}(\mathcal{O}) \times \mathbf{C}^{n+k} .
$$

For any $a \in \mathbf{C} \otimes T(M)$, we have (note that we now use $a(y)$ to mean the action of the vector $a$ on the function $y$ and we employ the summation convention)

$$
F_{*}(a)=a\left(f_{j}\right) \partial_{x_{j}}+a\left(y_{k}\right) \partial_{z_{k}}+a\left(\overline{y_{k}}\right) \partial_{\overline{z_{k}}}
$$

and

It follows that

$$
\mu F_{*}=a\left(y_{k}\right) \partial_{z_{k}}
$$

$$
B_{1}=\operatorname{ker} \mu F_{*}=\left\{a \in \mathbf{C} \otimes T(M): a\left(y_{k}\right)=0 \text { for } k=1, \ldots, n+1\right\}
$$

and this is involutive.

\section{Lemma 4.3.}

$$
B_{1} \cap \overline{B_{1}}=\{0\} .
$$

Proof. This is a consequence of the second condition of the Theorem.

Thus $B_{1}$ is a CR structure homotopic to $B$. (The proof of Theorem 4.1 will show that this homotopy is through almost CR structures.)

Finally, we need the map $\Psi$.

Lemma 4.4. If $(\mathbf{C} \otimes T(M)) / B$ is isomorphic to the Haefliger normal bundle $\nu$ then there exists a surjective bundle map

$$
\Psi:\left.\mathbf{C} \otimes T(M) \rightarrow T_{f}^{1,0}\right|_{M}
$$

with $B=\operatorname{ker} \Psi$.

Proof. We have

$$
\mathbf{C} \otimes T(M) \rightarrow(\mathbf{C} \otimes T(M)) /\left.B \stackrel{\alpha}{\rightarrow} \nu \stackrel{\beta}{\rightarrow} T_{f}^{1,0}\right|_{M}
$$

Since $\alpha$ and $\beta$ are isomorphisms, the composite map $\Psi$ is surjective and has kernel $B$. 


\section{An APPlichtion OF THE H-PRINCIPLE}

The general set-up is to define a subset $\mathcal{R} \subset J^{1}(M, X)$, called a relation, with the property that $j^{1}(F) \in \mathcal{R}$ implies that $F$ satisfies the conclusions of Theorem 4.1. See [7, 9], 12 for discussions of the h-principle.

Again choosing local coordinates $x \in \mathbf{R}^{2 n+k}$ for $M, y \in \mathbf{R}^{2 n+k}$, and $z \in \mathbf{C}^{n+k}$ for $\nu$ we see that

$$
\mu F_{*}: \mathbf{C} \otimes T(M) \rightarrow T_{f}^{1,0}
$$

is surjective if the rank of the matrix

$$
\left(\frac{\partial z_{i}}{\partial x_{j}}\right), \quad 1 \leq i \leq n+k \text { and } 1 \leq j \leq 2 n+k
$$

is maximal (i.e., equal to $n+k$ ) and that

$$
\mu F_{*}: T(M) \rightarrow T_{f}^{1,0}
$$

is injective if the rank of the $(2 n+2 k) \times(2 n+k)$ matrix

$$
\left(\begin{array}{c}
\frac{\partial z_{i}}{\partial x_{j}} \\
\frac{\partial \overline{z_{i}}}{\partial x_{j}}
\end{array}\right)
$$

is also maximal.

So we define our relation by

$$
\mathcal{R}=\left\{\left(p, c, a_{i}^{j}\right): \operatorname{rank}(a)=n+k, \quad \operatorname{rank}\left(\begin{array}{c}
a \\
\bar{a}
\end{array}\right)=2 n+k\right\}
$$

where, as above

$$
1 \leq i \leq n+k, \quad 1 \leq j \leq 2 n+k .
$$

We claim that $\mathcal{R}$ is an open and ample subset of $J^{1}(M, X)$. For the definition of ample see any of the above cited works or [13] for the proof of the claim see [13], pages 157-159. It is a consequence of the h-principle that the desired $F$ exists provided we can find a section

$$
\sigma: M \rightarrow \mathcal{R}
$$

That is, the existence of a formal solution implies the existence of a genuine solution.

To see that we do have a formal solution, we identify a bundle map

$$
\sigma: \mathbf{C} \otimes T(M) \rightarrow T_{f}^{1,0}
$$

with a section

$$
\tilde{\sigma}: M \rightarrow J^{1}(M, X)
$$

by writing

and then setting

$$
\sigma\left(\left.\partial_{x_{j}}\right|_{p}\right)=\left.h_{i}^{j} \partial_{z_{i}}\right|_{\sigma(p)}
$$

$$
\tilde{\sigma}(p)=\left(p, \sigma(p), h_{i}^{j}\right) .
$$

As long as $\sigma$ is surjective and its kernel satisfies $B \cap \bar{B}=\{0\}$, so

$$
\sigma: T(M) \rightarrow T_{f}^{1,0}
$$

is injective, $\tilde{\sigma}$ is a formal solution,

$$
\tilde{\sigma}: M \rightarrow \mathcal{R} \subset J^{1}(M, X) .
$$

In this context, Lemma 4.4 asserts the existence of a formal solution. 
The h-principle asserts more than the existence of a genuine solution. In fact, the genuine solution is obtained by a deformation

$$
\sigma_{t}: M \rightarrow \mathcal{R} \quad 0 \leq t \leq 1
$$

such that $\sigma_{0}=\tilde{\sigma}$ and $\sigma_{1}=j^{1}(F)$. Now set

$$
B_{t}=\operatorname{ker} \mu \sigma_{t} \text {. }
$$

We have rank $B_{t}=n$ and $B_{t} \cap \overline{B_{t}}=\{0\}$ for all $t$, and moreover $B_{1}$ is involutive.

Thus $B_{t}$ represents a deformation of $B$ through almost CR structures to a true CR structure.

Finally, once we have $F: M \rightarrow X$ we may deform $F$, maintaining the open conditions on the ranks of the matrices, to a $C^{\omega}$ map.

The proof of Theorem 3.2 is complete.

\section{Classifying spaces}

We first note that the definition of a Haefliger CR structure makes sense (for fixed $n$ and $k$ ) if $M$ is merely a topological space. To specify $n$ and $k$ we speak of an $(n, k)$ Haefliger CR structure. (Actually, only $n+k$ appears in the definition, so we occasionally speak of an $(n+k)$ Haefliger structure.) Also if $M$ and $N$ are topological spaces and $N$ has an $(n, k)$ Haefliger CR structure then any continuous map $\phi: M \rightarrow N$ induces an $(n, k)$ Haefliger CR structure on $M$.

Definition 3. Two $(n, k)$ Haefliger $C R$ structures on $N$ are homotopic if there exists an $(n, k)$ Haefliger $C R$ structure on $N \times I$ which pulls back to the given structures on $N \times\{0\}$ and $N \times\{1\}$.

Definition 4. A topological space $\mathcal{B}_{n, k}$ with an $(n, k)$ Haefliger $C R$ structure is a classifying space for $(n, k)$ Haefliger $C R$ structures if

- Every $(n, k)$ Haefliger $C R$ structure on a manifold $N$ is induced by a continuous map $\phi: N \rightarrow \mathcal{B}_{n, k}$.

- Two $(n, k)$ Haefliger $C R$ structures on $N$ are homotopic if and only if the corresponding continuous maps into $\mathcal{B}_{n, k}$ are homotopic.

The associated $(n+k)$-dimensional normal bundle over $\mathcal{B}_{n, k}$ is denoted by $\nu_{n, k}$.

Theorem 6.1. There exists a classifying space for $(n, k)$ Haefliger $C R$ structures.

This result is a particular case of the existence of classifying spaces for topological groupoid structures (as proved by Haefliger [10, [11] and others - see [16, page 48] or [18, page 312] for expository accounts). Indeed, these are precisely the classifying spaces for complex structures studied by Landweber 14 and by Adachi [1] and usually denoted by $B \Gamma_{q}^{\mathbf{C}}$. In the present case, the codimension $q$ is taken to be

$n+k$. Here $\Gamma_{q}^{\mathbf{C}}$ denotes the topological groupoid of germs of biholomorphisms between open subsets of $\mathbf{C}^{q}$.

\section{THE LIFTING PROBLEM}

We have started with a complex subbundle $B \subset \mathbf{C} \otimes T(M)$ and have set $\nu=$ $(\mathbf{C} \otimes T(M)) / B$. Using a Hermitian metric on $\mathbf{C} \otimes T(M)$ we may also consider $\nu$ as a subbundle of $\mathbf{C} \otimes T(M)$ complementary to $B$ in $\mathbf{C} \otimes T(M)$. Our aim is to show that the subbundle $\nu \subset \mathbf{C} \otimes T(M)$ of rank $n+k$ is the normal bundle of some $(n+k)$ Haefliger structure on $M$. Since bundles over $M$ are classified by homotopy 
classes of maps of $M$ into $B G L(n+k)$ (we denote by $G L(m)$ the complex general linear group $G L(m, \mathbf{C})$ ) and Haefliger structures are classified by homotopy classes of maps of $M$ into $\mathcal{B}_{n, k}$, the following set-up is natural. For any complex vector bundle $\xi$ of rank $m$ over a paracompact space $X$, let $\mathrm{cl}(\xi): X \rightarrow B G L(m)$ denote a classifying map for $\xi$. Thus we have the commutative diagram

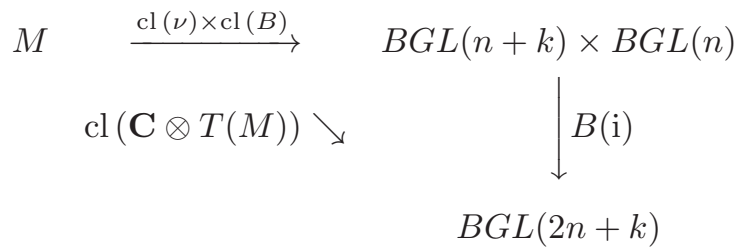

where i denotes the inclusion of $G L(n+k) \times G L(n)$ into $G L(2 n+k)$ by means of block matrices and $B(\mathrm{i})$ is the map of classifying spaces induced by this inclusion.

As above, let $\nu_{n, k}$ denote the normal bundle on the classifying space $\mathcal{B}_{n, k}$. Note that $\nu_{n, k}$ defines a map $\mathrm{cl}\left(\nu_{n, k}\right): \mathcal{B}_{n, k} \rightarrow B G L(n+k)$, unique up to homotopy.

Theorem 7.1. If

$$
H_{p}\left(M^{2 n+k} ; \mathbf{Z}\right)=0 \text { for } p \geq n+k+1
$$

then there exists a lift

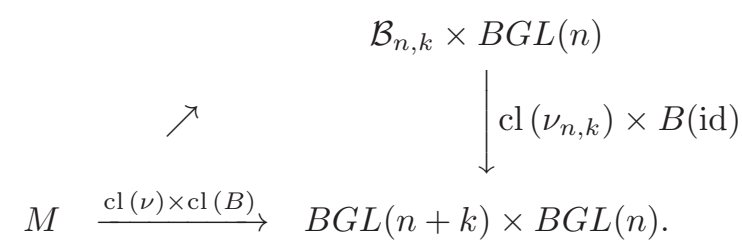

Proof. The homotopy fiber $\mathcal{F}=\mathcal{F}_{n, k}$ of the map cl $\left(\nu_{n, k}\right): \mathcal{B}_{n, k} \rightarrow B G L(n+$ $k$ ) is $(n+k)$-connected (cf. [1] and [14], although the latter paper needs to be supplemented by Gromov's h-principle for totally real immersions, as given in [8] and [9]). Since the obstructions to lifting a map

$$
M \rightarrow B G L(n+k) \times B G L(n)
$$

lie in $H^{j+1}\left(M ;\left\{\pi_{j}(\mathcal{F})\right\}\right)$ for the local coefficient system $\left\{\pi_{j}(\mathcal{F})\right\}$, and since $\pi_{j}(\mathcal{F})=$ 0 for $0 \leq j \leq n+k$, the nonzero obstructions can lie only in $H^{j+1}\left(M ;\left\{\pi_{j}(\mathcal{F})\right\}\right)$ for $j \geq n+k+1$. (For discussion of local coefficient systems, also known as bundles of coefficients or systems of local groups, we refer to Steenrod's book [23] on fiber bundles, or to his paper 22]. For a more recent account dealing with fibrations, see G. Whitehead's book [24, Chapter VI].) Since complex general linear groups are connected, their classifying spaces are simply connected, and so the map $\operatorname{cl}\left(\nu_{n, k}\right): \mathcal{B}_{n, k} \rightarrow B G L(n+k)$, which we view as a fibration, has simple local coefficient systems in the sense of 22. This means that there is a unique isomorphism between the homotopy groups of any two fibers. It follows that the local coefficient systems over $M$ appearing here are also simple. As a result, the cohomology groups here are ordinary cohomology groups and we can apply the usual universal coefficient theorem which expresses cohomology in terms of integral homology (note that homotopy groups beyond the first one are abelian and so the 
universal coefficient theorem applies). Hence if (7.1) holds then by the universal coefficient theorem we also have

$$
H^{j+1}\left(M ;\left\{\pi_{j}(\mathcal{F})\right\}\right)=0 \text { for } j \geq n+k+1
$$

and so every map (7.2) lifts to a map

$$
M \rightarrow \mathcal{B}_{n, k} \times B G L(n) .
$$

Remark 2. The hypothesis of Theorem 7.1 can be slightly weakened, Namely, it suffices to require that the integral homology groups in (7.1) vanish for $p \geq n+k+2$, and be free abelian for $p=n+k+1$. In particular, this weakened hypothesis is satisfied if the manifold $M^{2 n+k}$ has the homotopy type of a $C W$ complex of dimension at most $n+k+1$. This last condition is satisfied if $M^{2 n+k}$ has the homotopy type of an open $(n+k+2)$-manifold, which follows from [21, Lemma 1]. In short, if $M^{2 n+k}$ is homotopic to an open $(n+k+2)$ manifold, then the lift exists and Theorem 1.1 holds in this slightly more general case.

\section{THE THEOREM}

Theorem 8.1. If $M^{2 n+k}$ satisfies the condition (7.1) of Theorem 7.1 then every smooth almost $C R$ structure of codimension $k$ on $M$ is homotopic to a $C^{\omega} C R$ structure of codimension $k$. In particular, every $C^{\infty}$ CR structure may be deformed to a $C^{\omega}$ CR structure.

Proof. The lifting $M \rightarrow \mathcal{B}_{n, k} \times B G L(n)$ of Theorem 7.1 shows that $\nu \oplus B$ is isomorphic to some bundle $\nu^{0} \oplus B^{0}$ with $\nu^{0}$ the normal bundle of some $(n, k)$ Haefliger structure on $M$ and $B^{0}$ a complex bundle of rank $n$. The map $\operatorname{cl}(\nu) \times \operatorname{cl}(B)$ is homotopic to the map $\operatorname{cl}(\mathbf{C} \otimes T(M))$ and so, using the fact that the diagram is commutative we see that

$$
\nu^{0} \oplus B^{0} \cong \mathbf{C} \otimes T(M)
$$

In particular, $\nu^{0}$ and $B^{0}$ can be identified with subbundles of $\mathbf{C} \otimes T(M)$. Further, since the map $\operatorname{cl}\left(\nu_{n, k}\right) \times B(\mathrm{id})$ is the identity on the $B G L(n)$ factor, we have that $\nu$ is isomorphic to $\nu^{0}$. Thus Theorem 3.2 may be applied to show that $B$ may be deformed to some CR structure. Further, since this CR structure is induced by a map $F: M \rightarrow X$, as in Theorem 4.1, the map $F$ itself may be deformed to yield a $C^{\omega}$ CR structure. This last step just uses that the real analytic functions are dense in the set of smooth functions.

Note that the requirement that the integral homology of $M$ vanish in high dimensions excludes compact manifolds, provided that $n>1$. In the case $n=1$ the theorem holds for a trivial reason: Each almost CR structure with $n=1$ is automatically involutive.

Remark 3. This theorem also holds for $k=0$, as in [1] and [14, but our proof does not. The reason for this, in a very similar case, is explained in 13 .

In view of the analogy between foliations and involutive structures it is natural to try to place into this new context Bott's necessary condition for a subbundle of $T M$ to be homotopic to a foliation. See [3, 4, or [5] for Bott's original argument and some of its further consequences. 


\section{REFERENCES}

[1] M. Adachi, Embeddings and immersions. Translations of Mathematical Monographs, 124. American Mathematical Society, Providence, RI, 1993.

[2] M. S. Baouendi, P. Ebenfelt and L. P. Rothschild, Real submanifolds in complex space and their mappings. Princeton Mathematical Series, 47. Princeton University Press, Princeton, NJ, 1999.

[3] R. Bott, On a topological obstruction to integrability. Global Analysis (Proc. Sympos. Pure Math., Vol. XVI, 1968), pp. 127-131. Amer. Math. Soc., Providence, R.I., 1970.

[4] R. Bott, On topological obstructions to integrability. Actes du Congrs International des Mathmaticiens (Nice, 1970), Tome 1, pp. 27-36. Gauthier-Villars, Paris, 1971.

[5] R. Bott, Lectures on characteristic classes and foliations. Lectures on algebraic and differential topology, pp. 1-94. Lecture Notes in Math., Vol. 279, Springer, Berlin, 1972.

[6] B. Eckmann and A. Frölicher, Sur l'intégrabilité des structures presque complexes, C. R. Acad. Sci. Paris 232 (1951), 2284-2286.

[7] Y. Eliashberg and N. Mishachev, Introduction to the h-principle. Graduate Studies in Mathematics, 48. American Mathematical Society, Providence, RI, 2002.

[8] M. L. Gromov, Convex integration of differential relations. I. (Russian) Izv. Akad. Nauk SSSR Ser. Mat. 37 (1973), 329-343; English translation in Math. USSR Izvestiya 7 (1973), 329-343.

[9] M. Gromov, Partial differential relations. Ergebnisse der Mathematik und ihrer Grenzgebiete (3) [Results in Mathematics and Related Areas (3)], 9. Springer-Verlag, Berlin, 1986.

[10] A. Haefliger, Feuilletages sur les variétés ouvertes, Topology 9 (1970), 183-194.

[11] A. Haefliger, Homotopy and integrability. Manifolds-Amsterdam 1970, pp. 133-163, Lecture Notes in Mathematics, Vol. 197. Springer, Berlin, 1971.

[12] H. Jacobowitz, Convex integration and the h-principle. Lecture Notes Series, 55. Seoul National University, Seoul, 2011.

[13] H. Jacobowitz and P. Landweber, Manifolds admitting generic immersions into $\mathbf{C}^{n}$, Asian J. Math. 11 (2007), 151-165.

[14] P. S. Landweber, Complex structures on open manifolds, Topology 13 (1974), 69-75.

[15] H. B. Lawson, Jr., Foliations, Bull. Amer. Math. Soc. 80 (1974), 369-418.

[16] H. B. Lawson, Jr., The quantitative theory of foliations. Conference Board of the Mathematical Sciences Regional Conference Series in Mathematics, No. 27. American Mathematical Society, Providence, RI, 1977.

[17] P. Libermann, Problèmes d'quivalence relatifs à une structure presque complexe sur une variété à quatre dimensions, Acad. Roy. Belgique. Bull. Cl. Sci. (5) 36 (1950), 742-755.

[18] J. Milnor, Foliations and foliated vector bundles, in Collected papers of John Milnor, IV. Homotopy, homology and manifolds. Edited by John McCleary, pp. 279-320. American Mathematical Society, Providence, RI, 2009.

[19] A. Newlander and L. Nirenberg, Complex analytic coordinates in almost complex manifolds, Annals of Math. (2) 65 (1957), 391-404.

[20] L. Nirenberg, Lectures on linear partial differential equations. Conference Board of the Mathematical Sciences Regional Conference Series in Mathematics, No. 17. American Mathematical Society, Providence, R.I., 1973.

[21] A. Phillips, Submersions of open manifolds, Topology 6 (1967), 171-206.

[22] N. Steenrod, Homology with local coefficients, Annals of Math. (2) 44 (1943), 610-627.

[23] N. Steenrod, The topology of fibre bundles. Princeton Mathematical Series, 14. Princeton University Press, Princeton, NJ, 1951.

[24] G. W. Whitehead, Elements of homotopy theory. Graduate Texts in Mathematics, 61. Springer-Verlag, New York, 1978.

Department of Mathematical Sciences, Rutgers University, Camden, New Jersey, USA

E-mail address: jacobowi@camden.rutgers.edu

Department of Mathematics, Rutgers University, Piscataway, NJ 08854, USA

E-mail address: landwebe@math.rutgers.edu 